\title{
Analyzing Multiple Vector Autoregressions through Matrix-variate Normal Distribution with Two \\ Covariance Matrices
}

\author{
Nuttanan Wichitaksorn*
}

August 13, 2018

\begin{abstract}
This paper proposes a new approach to analyze multiple vector autoregressive (VAR) models that render us a newly constructed matrix autoregressive (MtAR) model based on a matrix-variate normal distribution with two covariance matrices. The MtAR is a generalization of VAR models where the two covariance matrices allow the extension of MtAR to a structural MtAR analysis. The proposed MtAR can also incorporate different lag orders across VAR systems that provide more flexibility to the model. The estimation results from a simulation study and an empirical study on macroeconomic application show favorable performance of our proposed models and method.

Keywords: Markov chain Monte Carlo, Multivariate analysis, Matrix-variate normal distribution, Autoregression
\end{abstract}

* Mathematical Sciences Department, Auckland University of Technology, Auckland 1010, New Zealand; School of Mathematics and Statistics, University of Canterbury, Christchurch 8140, New Zealand; and Thailand Development Research Institute, Bangkok 10310, Thailand, Email: nuttanan.wichitaksorn@aut.ac.nz 


\section{Introduction}

Vector autoregressive (VAR) models have been well studied and widely used in many macroeconomic applications. Various forms have been analyzed and result in a long list of the VAR literature. Among others, Lütkepohl (2007), Koop and Korobilis (2009), Del Negro and Schorfheide (2011), and Karlsson (2013) provide comprehensive overview on various VAR models and their formulation and implementation. Extension of the VAR models cover a wide range of applications, e.g., dynamic factor, time varying parameters, and dynamic stochastic general equilibrium models.

A more meaningful extension of the VAR is its structural model or SVAR. Originated by Sim (1980), SVAR models have been proven useful in macroeconomic analysis, especially in investigating the policy effects and forecasting economic time series; see, among others, Watson (1994), Christiano et al. (1999), Stock and Watson (2001), and Christiano et al. (2005), for various implementations and applications on the SVAR. One major issue that has been proposed and attempted on the SVAR is its identification; see Rubio-Ramírez et al. (2010), among others.

The estimation of VAR and SVAR has been well performed within the Bayesian framework through Markov chain Monte Carlo (MCMC) methods; see Sims and Zha (1998), Del Negro and Schorfheide (2011), among others for the implementation of the VAR and SVAR models using Bayesian MCMC methods.

One recent extension that has received much attention, especially for the transmission of shocks, is the multicountry VAR models. This group of models attempts to analyze the transmission effects across the units (countries) and the lagged intertemporal effects across (time) series through a covariance matrix; see, among others, Elliot and Fatás (1996), Canova and Marrinan (1998), Canova and De Nicolo (2000), Del

Negro and Obiols (2001), Canova and Ciccarelli (2009), Bańbura et al. (2010), and Favero (2013), for works on multicountry VAR models. Note that the units of interest do not need to be countries but can also be other objects such as states and agents. 
In general, the analysis of a multicountry VAR model is made through a single, large VAR system, even each country has the same structure (type of variables) and dimension (number of dependent variables). The dependence structure is then assessed using a covariance matrix that combines the across-country and across-variable effects. However, the analysis of a multicountry VAR model with the same structure and dimension can actually be made through a matrix system by stacking together each country VAR model. Hence, the across-country (across-unit) and across-variable (across-series) effects can be simultaneously and separately analyzed.

In this paper, we extend the analysis of VAR models by proposing a new approach to analyze multiple vector autoregressive (VAR) models that result in a newly constructed matrix autoregressive (MtAR) model. This extension enables us to conveniently analyze the multiple VAR systems in one setting. It also allows us to efficiently estimate and generalize the dependence (covariance) structure of the model. The construction of the MtAR is based on a matrix-variate normal distribution with two covariance matrices. The proposed MtAR is a generalization of the multivariate VAR models where the two covariance matrices allow the extension of MtAR to a structural MtAR (SMtAR) analysis. The proposed model is useful to a structural analysis, especially for macroeconomic application where we can analyze the dependence structure across units (e.g. countries) and across series (e.g. economic time series) through two covariance matrices in one go.

One major advantage of the SMtAR is that it can incorporate large VAR systems where their dependence structure is summarized through two covariance matrices. Hence, stacking the series of interest into matrix and analyzing it through the SMtAR render us the across-unit and across-series covariance matrices. In addition, working through the Kronecker product of two covariance matrices can return the large covariance matrix, that shows the interdependencies across units and series, as in the usual multicountry VAR models. However, the opposite is not true: with the unknown two covariance matrices, we cannot recover the two covariance matrices from the large 
covariance matrix. Also, our model is flexible enough to incorporate different lags for different VAR systems.

Since the construction of the proposed SMtAR is based on a (matrix-variate normal) distribution, the Bayesian parametric approach is a good candidate for the estimation. Under some assumptions, we show that the model implementation is straightforward and can be easily made using the MCMC. In addition, we find from a simulation study that the MCMC estimation returns favorable results and perform better than the maximum likelihood (ML) estimation and the feasible generalized least squares (FGLS).

The organization of this paper is as follows: Section 2 briefly introduces the matrixvariate normal distribution and also illustrates the formulation of the MtAR and SMtAR models. Section 3 presents the MCMC estimation. Section 4 performs a simulation study. Section 5 shows the applicability of the proposed models through an empirical analysis. Section 6 provides the concluding remarks.

\section{Model}

\subsection{Matrix-variate normal distribution}

According to Dawid (1981) and Gupta and Nagar (1999, pp.55-56), a $d \times k$ matrix $\mathbf{Y}$ is said to be matrix-variate normal random variable or $\mathbf{Y} \sim M N_{d, k}(\mathbf{M}, \boldsymbol{\Sigma}, \boldsymbol{\Psi})$ if its probability density function is given by

$$
f(\mathbf{Y} \mid \mathbf{M}, \boldsymbol{\Sigma}, \boldsymbol{\Psi})=(2 \pi)^{-\frac{d k}{2}}|\boldsymbol{\Sigma}|^{-\frac{k}{2}}|\Psi|^{-\frac{d}{2}} \exp \left\{-\frac{1}{2} \operatorname{tr}\left[\boldsymbol{\Psi}^{-1}(\mathbf{Y}-\mathbf{M})^{\prime} \boldsymbol{\Sigma}^{-1}(\mathbf{Y}-\mathbf{M})\right]\right\}
$$

where $\mathbf{M}$ is the $d \times k$ mean matrix, $\boldsymbol{\Sigma}$ is the $d \times d$ across-row covariance matrix, $\Psi$ is the $k \times k$ across-column covariance matrix, and $\operatorname{tr}$ denotes the matrix trace. If we vectorize the random matrix $\mathbf{Y}$, we get $\operatorname{vec}(\mathbf{Y}) \sim N_{d k}(\operatorname{vec}(\mathbf{M}), \mathbf{\Psi} \otimes \mathbf{\Sigma})$, which is a $d k$-dimensional multivariate normal random variable with $d k \times 1$ mean vector $v e c(\mathbf{M})$ 
and $d k \times d k$ covariance matrix $\Psi \otimes \Sigma$; see proof in Gupta and Nagar (1999, pp.55-56).

Hence, the pdf of $\operatorname{vec}(\mathbf{Y})$ is given by

$f(\operatorname{vec}(\mathbf{Y}) \mid \operatorname{vec}(\mathbf{M}), \mathbf{\Psi} \otimes \boldsymbol{\Sigma})=(2 \pi)^{-\frac{d k}{2}}|\mathbf{\Psi} \otimes \boldsymbol{\Sigma}|^{-\frac{d k}{2}} \exp \left\{-\frac{1}{2}\left[\operatorname{vec}(\mathbf{Y}-\mathbf{M})^{\prime}(\mathbf{\Psi} \otimes \boldsymbol{\Sigma})^{-1} \operatorname{vec}(\mathbf{Y}-\mathbf{M})\right]\right\}$

which is equivalent to (1). Note that $\operatorname{vec}(\mathbf{Y}-\mathbf{M})=\operatorname{vec}(\mathbf{Y})-\operatorname{vec}(\mathbf{M})$; see e.g. Exercise 10.16 in Abadir and Magnus (2005, p.281). Let $\boldsymbol{\Omega} \equiv \boldsymbol{\Psi} \otimes \boldsymbol{\Sigma}$. Following the pdf in (2), we can then generate the random vector $\operatorname{vec}(\mathbf{Y})$ from $N_{d k}(\operatorname{vec}(\mathbf{M}), \boldsymbol{\Omega})$. It is worth noting here that we can obtain $\Omega$ from $\boldsymbol{\Psi} \otimes \boldsymbol{\Sigma}$ but we cannot decompose the unknown $\boldsymbol{\Psi}$ and $\boldsymbol{\Sigma}$ from $\boldsymbol{\Omega}$; see, e.g., Liu (2012).

\subsection{Matrix autoregressive (MtAR) model}

Let us consider a reduced-form vector autoregressive model with order $p$ or $\operatorname{VAR}(p)$ given by

$$
y_{t}=c+A_{1} y_{t-1}+A_{2} y_{t-2}+\cdots+A_{p} y_{t-p}+u_{t}
$$

for $t=1, \ldots, T$ where $y_{t}=\left(y_{1 t}, \ldots, y_{d t}\right)^{\prime}$ is a $d \times 1$ vector of present observations, $A_{i}$ are $d \times d$ matrices of autoregressive coefficients for $i=1, \ldots, p, c$ is a $d \times 1$ vector of constants, and $u_{t}$ is a $d \times 1$ iid normal random vector with mean vector $\mathbf{0}$ and positive-definite covariance matrix $\boldsymbol{\Sigma}$ or $u_{t} \stackrel{i i d}{\sim} N(\mathbf{0}, \boldsymbol{\Sigma})$. The reduced-form VAR $(p)$ model in $(3)$ can be written in the seemingly unrelated regression (SUR) form as

$$
y_{t}=B x_{t}+u_{t}
$$

where $B=\left[c A_{1} \cdots A_{p}\right]$ is the $d \times(p d+1)$ matrix of regression coefficients and $x_{t}=$ $\left(1, y_{1, t-1}, \ldots, y_{d, t-1}, \ldots, y_{1, t-p}, \ldots, y_{d, t-p}\right)^{\prime}$ is the $(p d+1) \times 1$ vector of covariates. 
The basic idea behind the construction of an MtAR model is stacking together all data into matrices and representing the model in terms of SUR where the $k$ columns of model errors $\left[u_{t}^{1} \cdots u_{t}^{k}\right]$ are stacked as a matrix and assumed to follow the matrixvariate normal distribution described in Section 2.1. That is, for $m=1, \ldots, k$ the contemporaneous or across-row covariance matrix is $\boldsymbol{\Sigma}$ and for $j=1, \ldots, d$ the acrossobject or across-column covariance matrix is $\boldsymbol{\Psi}$. Intuitively, $\boldsymbol{\Sigma}$ summarizes the acrossrow covariance structure while $\boldsymbol{\Psi}$ provides similar information across columns.

Consider an MAR(1) model where two VAR(1) models are stacked together and written in a generic SUR form as

$$
\mathbf{Y}_{t}=\mathbf{B X}_{t}+\mathbf{U}_{t}
$$

where $\mathbf{Y}_{t}=\left[\begin{array}{ll}y_{t}^{1} & y_{t}^{2}\end{array}\right]$ is the $d \times 2$ matrix of present observations, $\mathbf{B}=\left[\begin{array}{ll}B^{1} & B^{2}\end{array}\right]$ is the $d \times 2(d+1)$ matrix of regression coefficients, $\mathbf{X}_{t}$ is the $2(d+1) \times 2$ matrix of covariates that $x_{t}^{1}$ and $x_{t}^{2}$ are stacked as

$$
\mathbf{X}_{t}=\left[\begin{array}{cc}
1 & 0 \\
y_{1, t-1}^{1} & 0 \\
\vdots & \vdots \\
y_{d, t-1}^{1} & 0 \\
0 & 1 \\
0 & y_{1, t-1}^{2} \\
\vdots & \vdots \\
0 & y_{d, t-1}^{2}
\end{array}\right],
$$

and $\mathbf{U}_{t}=\left[u_{t}^{1} u_{t}^{2}\right]$ is the $d \times 2$ matrix of model errors. If we stack the $k$ VAR models with the same lag order $p$, we can obtain the $\operatorname{MtAR}(p)$ model with $\mathbf{Y}_{t}=\left[y_{t}^{1} \cdots y_{t}^{k}\right]$, $\mathbf{B}=\left[B^{1} \cdots B^{k}\right], \mathbf{U}_{t}=\left[u_{t}^{1} \cdots u_{t}^{k}\right]$, and $x_{t}^{1} \cdots x_{t}^{k}$ are stacked into $\mathbf{X}_{t}$ in the same way as above. 
Our proposed MtAR model also allows the flexible modeling where different VAR systems can have different lag orders. Let $\mathbf{p}=\left[p^{1} \cdots p^{k}\right]$ denote a vector of lag orders. It follows that the $\operatorname{MtAR}(\mathbf{p})$ is the model with the matrix of regression coefficients $\mathbf{B}$ is a $d \times\left(d\left(p^{1}+\cdots+p^{k}\right)+k\right)$ matrix and the matrix of covariates $\mathbf{X}_{t}$ is a $\left(d\left(p^{1}+\cdots+p^{k}\right)+k\right) \times k$ matrix while $\mathbf{Y}_{t}$ and $\mathbf{U}_{t}$ are still a $d \times k$ matrix.

\subsection{Structural MtAR model}

The SUR representation of the MtAR in (5) can also be regarded as the reducedform MtAR. Since the error matrix $\mathbf{U}_{t}$ is assumed to follow a matrix-variate normal distribution with two covariance matrices $\boldsymbol{\Sigma}$ and $\boldsymbol{\Psi}$ or $\mathbf{U}_{t} \sim M N_{d, k}(\mathbf{0}, \boldsymbol{\Sigma}, \boldsymbol{\Psi})$ together with the restrictions on the two covariance matrices, the MtAR in (5) can be used for a structural analysis as in the case of SVAR and called SMtAR. For conciseness, we consider only the case where the SMtAR is exactly identified as in Theorem 4 of RubioRamirez et al. (2010), which states that if for every reduced-form parameter point there exists a unique structural parameter point on the real line such that the reduced-form parameter point is a function of structural parameter point, then the restrictions on the structural parameter matrix are triangular; see Rubio-Ramirez et al. (2010) and Karlsson (2013), among others, for more details on the identification of an SVAR and the restrictions on its covariance matrix.

The construction of SMtAR follows from the SVAR where in this case it is based on across-row and across-column SVAR systems. From (4), it follows that, for each column $m$, the reduced form of across-row SVAR system is given by

$$
y_{t}^{m}=B^{m} x_{t}^{m}+u_{t}^{m} \text {, }
$$

where $u_{t}^{m} \sim N_{d}(0, \boldsymbol{\Sigma})$. Let $\boldsymbol{\Sigma}=\left(A_{0} A_{0}^{\prime}\right)^{-1}$ where $A_{0}$ is a full-rank triangular matrix, which is called the contemporaneous coefficient matrix, $B^{m}=A_{0}^{-1} A^{m}$, and $u_{t}^{m}=$ $A_{0}^{-1} e_{t}^{m}$, where $e_{t}^{m} \sim N_{d}\left(0, I_{d}\right)$. The structural form of across-row SVAR system in 6 6 
can then be written as

$$
A_{0} y_{t}^{m}=A^{m} x_{t}^{m}+e_{t}^{m}
$$

Similarly, for each row $j$ the reduced form of across-column SVAR system is given by

$$
y_{j, t}^{\prime}=B_{j} x_{j, t}^{\prime}+u_{j, t}^{\prime}
$$

where $y_{j, t}=\left[y_{j, t}^{1} \cdots y_{j, t}^{k}\right]$ is the $1 \times k$ vector of present observations, $B_{j}=\left[c_{j}^{\prime} A_{j, 1}^{\prime} \cdots A_{j, p}^{\prime}\right]$ is the $k \times(p k+1)$ matrix of regression coefficients, $x_{j t}=\left[\begin{array}{lll}1 & y_{j, t-1}^{1} \cdots y_{j, t-p}^{k}\end{array}\right]$ is the $1 \times(p k+1)$ vector of covariates, and $u_{j t}^{\prime} \sim N_{k}(0, \boldsymbol{\Psi})$ is the $k \times 1$ vector of across-object errors. Let $\boldsymbol{\Psi}=\left(\Lambda_{0} \Lambda_{0}^{\prime}\right)^{-1}$ where $\Lambda_{0}$ is a full-rank triangular matrix, which is called the across-object coefficient matrix, $B_{j}=\Lambda_{0}^{-1} \Lambda_{j}$, and $u_{j, t}^{\prime}=\Lambda_{0}^{-1} e_{j, t}^{\prime}$ where $e_{j, t}^{\prime} \sim N_{k}\left(0, I_{k}\right)$. The structural form of across-column SVAR system in (7) can then be written as

$$
\Lambda_{0} y_{j, t}^{\prime}=\Lambda_{j} x_{j, t}^{\prime}+e_{j, t}^{\prime}
$$

Again, after stacking all data together, we can analyze the SMtAR through the reducedform SUR in (5) where we estimate the matrix of regression coefficients $\mathbf{B}$ and the two covariance matrices $\boldsymbol{\Sigma}$ and $\boldsymbol{\Psi}$, hence the contemporaneous and across-object coefficient matrices $A_{0}$ and $\Lambda_{0}$.

If we vectorize $\mathbf{Y}_{t}$ and $\mathbf{U}_{t}$ in (5) and stack $\mathbf{B}$ and $\mathbf{X}_{t}$ accordingly, we can obtain the following vectorized SMtAR model

$$
\operatorname{vec}\left(\mathbf{Y}_{t}\right)=\left(\mathbf{X}_{t}^{\prime} \otimes I_{d}\right) \operatorname{vec}(\mathbf{B})+\operatorname{vec}\left(\mathbf{U}_{t}\right)
$$

where $\operatorname{vec}\left(\mathbf{U}_{t}\right) \sim N_{d k}(0, \boldsymbol{\Omega})$ and $\boldsymbol{\Omega}=\boldsymbol{\Psi} \otimes \boldsymbol{\Sigma}$. Let $\boldsymbol{\Omega}=\left(V_{0} V_{0}^{\prime}\right)^{-1}$ where $V_{0}$ is a $d k \times$ $d k$ full-rank triangular matrix. It follows that $V_{0}$ is the matrix that indicates the contemporaneous and across-object effects. 
The extension of our proposed SMtAR can cover a wide range of specifications. Among others, we can allow the regression coefficients to be time varying, and if we vectorize the SMtAR as that in (8), the vectorized SMtAR can be analogous to the multi-country VAR model in Canova and Ciccarelli (2009). However, the analysis of SMtAR with time-varying coefficients is beyond the scope of this paper and will not be pursued here.

\section{Estimation}

This section shows the implementation of the SMtAR model where the model parameters are estimated using the Bayesian MCMC method. With the reduced-form SMtAR in (5), the model likelihood is then given by

$L\left(\mathbf{Y}_{1: T} \mid \mathbf{X}_{1: T}, \mathbf{B}, \boldsymbol{\Sigma}, \boldsymbol{\Psi}\right)=\prod_{t=1}^{T}(2 \pi)^{-\frac{d k}{2}}|\boldsymbol{\Sigma}|^{-\frac{k}{2}}|\boldsymbol{\Psi}|^{-\frac{d}{2}} \exp \left\{-\frac{1}{2} \operatorname{tr}\left[\boldsymbol{\Psi}^{-1}\left(\mathbf{Y}_{t}-\mathbf{B X}_{t}\right)^{\prime} \boldsymbol{\Sigma}^{-1}\left(\mathbf{Y}_{t}-\mathbf{B} \mathbf{X}_{t}\right)\right]\right\}$

Applying the independent conjugate priors to the tractable likelihood in (9), we can draw the model parameters using Gibbs sampler.

\subsection{Drawing matrix of regression coefficients (B)}

We apply the conjugate matrix-variate normal prior for the matrix of regression coefficients $\mathbf{B} \sim M N_{d, d q+k}\left(\mathbf{B}_{0}, \mathbf{C}_{0}, \mathbf{S}_{0}\right)$ where $q=\left(p^{1}+\cdots+p^{k}\right), \mathbf{B}_{0}, \mathbf{C}_{0}$, and $\mathbf{S}_{0}$ are hyperparameter matrices. Hence, the posterior density for $\mathbf{B}$ is given by

$$
\begin{aligned}
f\left(\mathbf{B} \mid \mathbf{B}_{0}, \mathbf{C}_{0}, \mathbf{S}_{0}, \mathbf{Y}_{1: T}, \mathbf{X}_{1: T}, \boldsymbol{\Sigma}, \boldsymbol{\Psi}\right) \propto & \exp \left\{-\frac{1}{2} \operatorname{tr}\left[\mathbf{S}_{0}^{-1}\left(\mathbf{B}-\mathbf{B}_{0}\right)^{\prime} \mathbf{C}_{0}^{-1}\left(\mathbf{B}-\mathbf{B}_{0}\right)\right]\right\} \\
& \times \exp \left\{-\frac{1}{2} \sum_{t=1}^{T} \operatorname{tr}\left[\mathbf{\Psi}^{-1}\left(\mathbf{Y}_{t}-\mathbf{B} \mathbf{X}_{t}\right)^{\prime} \boldsymbol{\Sigma}^{-1}\left(\mathbf{Y}_{t}-\mathbf{B} \mathbf{X}_{t}\right)\right]\right\}
\end{aligned}
$$


To facilitate the draw of $\mathbf{B}$ from its posterior density, we vectorize all matrices in (10). The vectorized version of the posterior density is given by

$$
\begin{aligned}
f(\operatorname{vec}(\mathbf{B}) \mid \cdot) \propto & \exp \left\{-\frac{1}{2} \operatorname{vec}\left(\mathbf{B}-\mathbf{B}_{0}\right)^{\prime}\left(\mathbf{S}_{0} \otimes \mathbf{C}_{0}\right)^{-1} \operatorname{vec}\left(\mathbf{B}-\mathbf{B}_{0}\right)\right\} \\
& \times \exp \left\{-\frac{1}{2} \sum_{t=1}^{T} \operatorname{vec}\left(\mathbf{Y}_{t}-\mathbf{B X}_{t}\right)^{\prime}(\mathbf{\Psi} \otimes \mathbf{\Sigma})^{-1} \operatorname{vec}\left(\mathbf{Y}_{t}-\mathbf{B X}_{t}\right)\right\} .
\end{aligned}
$$

Let $\mathbf{Z}_{0}=\mathbf{S}_{0} \otimes \mathbf{C}_{0}, \boldsymbol{\Omega}=\mathbf{\Psi} \otimes \boldsymbol{\Sigma}$, and $\mathbf{W}_{t}=\mathbf{X}_{t}^{\prime} \otimes I_{d}$. It follows that

$$
\begin{aligned}
& f(\operatorname{vec}(\mathbf{B}) \mid \cdot) \propto \exp \left\{-\frac{1}{2}\left[\operatorname{vec}(\mathbf{B})^{\prime}\left(\mathbf{Z}_{0}^{-1}+\sum_{t=1}^{T} \mathbf{W}_{t}^{\prime} \boldsymbol{\Omega}^{-1} \mathbf{W}_{t}\right) \operatorname{vec}(\mathbf{B})\right.\right. \\
& \left.\left.-2 \operatorname{vec}(\mathbf{B})^{\prime}\left(\mathbf{Z}_{0}^{-1} \operatorname{vec}\left(\mathbf{B}_{0}\right)+\sum_{t=1}^{T} \mathbf{W}_{t}^{\prime} \boldsymbol{\Omega}^{-1} \operatorname{vec}\left(\mathbf{Y}_{t}\right)\right)\right]\right\} \\
& \propto \exp \left\{-\frac{1}{2}(\operatorname{vec}(\mathbf{B})-\overline{\operatorname{vec}(\mathbf{B})})^{\prime} \mathbf{B}_{1}^{-1}(\operatorname{vec}(\mathbf{B})-\overline{\operatorname{vec}(\mathbf{B})})\right\} \\
& \propto \quad N_{d(d q+k)}\left(\overline{\operatorname{vec}(\mathbf{B})}, \mathbf{B}_{1}\right)
\end{aligned}
$$

where $\overline{\operatorname{vec}(\mathbf{B})}=\mathbf{B}_{1}\left(\mathbf{Z}_{0}^{-1} \operatorname{vec}\left(\mathbf{B}_{0}\right)+\sum_{t=1}^{T} \mathbf{W}_{t}^{\prime} \boldsymbol{\Omega}^{-1} \operatorname{vec}\left(\mathbf{Y}_{t}\right)\right)$ and $\mathbf{B}_{1}=\left(\mathbf{Z}_{0}^{-1}+\sum_{t=1}^{T} \mathbf{W}_{t}^{\prime} \boldsymbol{\Omega}^{-1} \mathbf{W}_{t}\right)^{-1}$. If the matrix $\mathbf{B}$ is large, we can divide it into sub-blocks and sample them accordingly.

One prominent prior that has been popularly applied to analyze the VAR models is the Minnesota prior; see Litterman (1980) and Doan et al. (1984), among others, for more details on the Minnesota prior. However in this paper, we find from the simulation study in Section 4 that the conjugate multivariate normal prior is sufficient to produce good estimation results for the SMtAR models.

\subsection{Drawing across-row covariance matrix $(\Sigma)$}

With the conjugate inverse-wishart prior for $\Sigma \sim I W_{d}\left(w_{0}, \mathbf{Q}_{0}\right)$ where $w_{0}$ and $\mathbf{Q}_{0}$ are, respectively, hyperparameter and hyperparameter matrix, we can obtain the following 
posterior density

$$
\begin{aligned}
f\left(\boldsymbol{\Sigma} \mid \mathbf{Q}_{0}, w_{0}, \mathbf{Y}_{1: T}, \mathbf{X}_{1: T}, \mathbf{B}, \mathbf{\Psi}\right) \propto & \left|\mathbf{Q}_{0}\right|^{\frac{w_{0}}{2}}|\boldsymbol{\Sigma}|^{\frac{-\left(w_{0}+d+1\right)}{2}} \exp \left\{-\frac{1}{2} \operatorname{tr}\left(\mathbf{Q}_{0} \boldsymbol{\Sigma}^{-1}\right)\right\} \\
& \times|\boldsymbol{\Sigma}|^{\frac{-(T k)}{2}} \exp \left\{-\frac{1}{2} \sum_{t=1}^{T} \operatorname{tr}\left[\mathbf{\Psi}^{-1}\left(\mathbf{Y}_{t}-\mathbf{B} \mathbf{X}_{t}\right)^{\prime} \boldsymbol{\Sigma}^{-1}\left(\mathbf{Y}_{t}-\mathbf{B X}_{t}\right)\right]\right\} \\
\propto & |\boldsymbol{\Sigma}|^{\frac{-\left(T k+w_{0}+d+1\right)}{2}} \\
& \times \exp \left\{-\frac{1}{2} \operatorname{tr}\left[\left(\mathbf{Q}_{0}+\sum_{t=1}^{T}\left(\mathbf{Y}_{t}-\mathbf{B X}_{t}\right) \mathbf{\Psi}^{-1}\left(\mathbf{Y}_{t}-\mathbf{B X}_{t}\right)^{\prime}\right) \boldsymbol{\Sigma}^{-1}\right]\right\} \\
\propto & I W_{d}\left(T k+w_{0},\left[\mathbf{Q}_{0}+\sum_{t=1}^{T}\left(\mathbf{Y}_{t}-\mathbf{B X}_{t}\right) \mathbf{\Psi}^{-1}\left(\mathbf{Y}_{t}-\mathbf{B X}_{t}\right)^{\prime}\right]\right) .
\end{aligned}
$$

\subsection{Drawing across-column covariance matrix $(\Psi)$}

Similar to the across-row covariance matrix, we can apply the conjugate inverse-wishart

prior for $\boldsymbol{\Psi} \sim I W_{k}\left(g_{0}, \mathbf{G}_{0}\right)$ where $g_{0}$ and $\mathbf{G}_{0}$ are, respectively, hyperparameter and hyperparameter matrix and obtain the following posterior density

$$
\begin{aligned}
f\left(\boldsymbol{\Psi} \mid \mathbf{G}_{0}, g_{0}, \mathbf{Y}_{1: T}, \mathbf{X}_{1: T}, \mathbf{B}, \boldsymbol{\Sigma}\right) \propto & \left|\mathbf{G}_{0}\right|^{\frac{g_{0}}{2}}|\boldsymbol{\Psi}|^{\frac{-\left(g_{0}+k+1\right)}{2}} \exp \left\{-\frac{1}{2} \operatorname{tr}\left(\mathbf{G}_{0} \mathbf{\Psi}^{-1}\right)\right\} \\
& \times|\boldsymbol{\Psi}|^{\frac{-(T d)}{2}} \exp \left\{-\frac{1}{2} \sum_{t=1}^{T} \operatorname{tr}\left[\boldsymbol{\Psi}^{-1}\left(\mathbf{Y}_{t}-\mathbf{B X}_{t}\right)^{\prime} \boldsymbol{\Sigma}^{-1}\left(\mathbf{Y}_{t}-\mathbf{B X}_{t}\right)\right]\right\} \\
\propto & |\boldsymbol{\Psi}|^{\frac{-\left(T d+g_{0}+k+1\right)}{2}} \\
& \times \exp \left\{-\frac{1}{2} \operatorname{tr}\left[\left(\mathbf{G}_{0}+\sum_{t=1}^{T}\left(\mathbf{Y}_{t}-\mathbf{B X}_{t}\right)^{\prime} \boldsymbol{\Sigma}^{-1}\left(\mathbf{Y}_{t}-\mathbf{B X}_{t}\right)\right) \mathbf{\Psi}^{-1}\right]\right\} \\
\propto & I W_{k}\left(T d+g_{0},\left[\mathbf{G}_{0}+\sum_{t=1}^{T}\left(\mathbf{Y}_{t}-\mathbf{B X}_{t}\right)^{\prime} \boldsymbol{\Sigma}^{-1}\left(\mathbf{Y}_{t}-\mathbf{B X}_{t}\right)\right]\right) .
\end{aligned}
$$

After obtaining $\boldsymbol{\Sigma}$ and $\boldsymbol{\Psi}$, it becomes straightforward to compute the matrices $A_{0}, \Lambda_{0}, \Omega$, and $V_{0}$. 


\section{Simulation Study}

In this section, we illustrate the implementation of SMtAR models using simulated data through several examples. Let $d \times k \operatorname{SMtAR}(p)$ denote the SMtAR model with $d$ rows and $k$ columns of dependent variables $\mathbf{Y}_{t}$ where each having the same lag order $p$ and let $d \times k \operatorname{SMtAR}(\mathbf{p})$ denote the $d$-row and $k$-column SMtAR model with each $k$ VAR system having different lag orders $\mathbf{p}=\left[p^{1} \cdots p^{k}\right]$. The examples below are $2 \times 2$ $\operatorname{SMtAR}(1), 2 \times 2 \operatorname{SMtAR}(2), 3 \times 4 \operatorname{SMtAR}(1), 3 \times 4 \operatorname{SMtAR}(3)$, and $3 \times 4 \operatorname{SMtAR}(\mathbf{p})$ where $\mathbf{p}=\left[\begin{array}{llll}1 & 2 & 3 & 1\end{array}\right]$. Note that in each example, given the true parameter values on the covariance matrices, the random errors are first generated from the matrix-variate normal distribution. Then, given the regression coefficients the data with 700 time observations are generated where the first 200 observations are discarded to ensure the stability and reliability of the series. As a result, we obtain the $T=500$ time observations for the analysis. See more details on the true parameter values of some selected models in Appendix A.

In the estimation, we use the ordinary least squares estimates as the starting value for the MCMC algorithm. (We also tried the different starting value, e.g., zero-valued parameters but found no effects on the results and performance.) We monitor the convergence draws by examining the trace plots. Based on the construction of the matrix-variate normal distribution, the parameter draws of two covariance matrices ( $\boldsymbol{\Sigma}$ and $\boldsymbol{\Psi}$ ) can be highly correlated and result in the computational inefficiency. We carefully determine the inefficiency of the parameter draws through the simulation inefficiency factor (SIF); see Chib (2008) for more details on the SIF.

To obtain the low SIFs, we have to vary the thin size and the number of iterations in different SMtAR model specifications. For example in $2 \times 2$ SMtAR (the lowest

dimension for SMtAR model) where the posterior draws of two covariance matrices are highly correlated, to get the low SIFs (i.e., around 2) we have to keep every $100^{\text {th }}$ draw or the thin size is 100 . While for $3 \times 4 \mathrm{SMtAR}$, the thin size of 10 is sufficient to return 
the low SIF values for the covariance parameters.

In order to assess the performance of our proposed models and method, we compare the MCMC results to those from least squares and ML estimation. The least squares method we use is the FGLS where the implementation is made on the vectorized model in (8); see Wooldridge (2001, Section 7.5), among others, for more details on the FGLS. However, by using the FGLS we cannot recover the unknown $\boldsymbol{\Sigma}$ and $\Psi$ matrices from the $\Omega$ as the relation from $\Omega$ to $\Sigma$ and $\boldsymbol{\Psi}$ is one to many. Hence for the sake of comparison, we also draw $\Omega$ using the MCMC where we apply the conjugate inverse wishart prior $\Omega \sim I W_{d k}\left(r_{0}, \mathbf{R}_{0}\right)$, where $r_{0}$ and $\mathbf{R}_{0}$ are hyperparameter and hyperparameter matrix, that results in the inverse wishart posterior $\boldsymbol{\Omega} \mid \cdot \sim I W_{d k}\left(T+r_{0},\left[\mathbf{R}_{0}+\operatorname{vec}\left(\mathbf{Y}_{t}-\mathbf{B X}_{t}\right) \operatorname{vec}\left(\mathbf{Y}_{t}-\mathbf{B} \mathbf{X}_{t}\right)^{\prime}\right]\right)$.

For the ML method, since our SMtAR is also a generalized regression model represented in the SUR form, as indicated in Greene (2003, pp.347-349) the ML estimator of regression coefficients matrix $\mathbf{B}$ is equivalent to the FGLS estimator. Hence for convenience, we use the $\hat{\mathbf{B}}$ obtained from FGLS as the ML estimates. Then, we apply the ML algorithm by Dutilleul (1999) to obtain the estimates of $\boldsymbol{\Sigma}$ and $\boldsymbol{\Psi}$. The estimation results obtained from the three methods are judged through the deviation from the true parameter value using the mean absolute deviation (MAD) and the mean absolute percentage error (MAPE).

$<$ Tables 1-2 about here $>$

Tables 1-2 summarizes the estimation results from the five SMtAR models in favor of the MCMC method. (See Appendix Tables A1-A3 for more detailed results from some models.) Note that the average standard errors (S.E.) and MAD are not available in some cases because the $\hat{\Sigma}, \hat{\Psi}$, and the S.E. of $\hat{\boldsymbol{\Omega}}$ are not estimated under the FGLS while the $\hat{\Omega}$ is not estimated under the MLE.

Among the three methods, the MCMC returns the lowest MADs and MAPEs in all models and parameters. The average S.E. from MCMC are lower in most models and 
parameters except for $\hat{\mathbf{B}}$ in $3 \times 4 \operatorname{SMtAR}(1)$ and $3 \times 4 \operatorname{SMtAR}(\mathbf{p})$ where the FGLS produces the lower average S.E. It is also worth noting that the MCMC provides the reasonably low SIF values. With these favourable simulation results from the MCMC, we then proceed to apply our proposed models and method to a real data example in the next section.

\section{Empirical Study}

This section illustrates the application of our model and method to macroeconomic and financial data. The data contain five quarterly series including change in interest rate, inflation rate, change in unemployment rate, real GDP growth rate, and change in share prices from the group of seven leading economies (G-7) including US, UK, Canada, France, Germany, Italy, and Japan that result in a $5 \times 7$ SMtAR model. (We select the G-7 in the study as the assumptions on same across-series covariance and same across-countries covariance can be attainable.) The structural analysis of this model helps illustrate and summarize how the economic and financial series affect each other and how the shocks transmit through the seven countries. The data from the third quarter of 1991 to the second quarter of 2013 leave us with 88 time observations for the analysis.

To determine the number of lags, we employ the likelihood ratio test (provided in MATLAB) for each of the seven VAR systems. The appropriate numbers of lags for the $5 \times 7$ SMtAR model are $\mathbf{p}=\left[\begin{array}{llllll}3 & 4 & 2 & 4 & 1 & 4\end{array}\right]$ for the G-7, respectively. From a single run of 6,000 MCMC iterations including 1,000 burn-ins and keeping every fifth draw, we obtain 1,000 posterior samples for inference. For conciseness, we report only the estimation results from two covariance matrices.

$<$ Tables 3 and 4 about here>

Tables 3 and 4 show the estimation results for the two covariance matrices and their structural-effect matrix that summarize the dependence structures among G-7. 
The posterior means and standard errors from the contemporaneous or across-series

coefficient matrix $\hat{A}_{0}$ in Table 3 reveal that only effect of inflation rate on change in share prices is negative and significantly different from zero. This is not surprising as it has always been observed and commonly known that higher inflation drives down the purchasing power and hence lowers the share prices.

It is worth noting here that, though statistically insignificant, most of parameters from $\hat{A}_{0}$ have the expected sign. There are only two parameters that their sign are not as expected; these are (1) the relationship between chage in real GDP and change in unemployment rate and (2) the relationship between change in real GDP and change in share prices. The estimated coefficient for change in real GDP and change in unemployment rate seems contradict to the Okun's law, which states that the increase in unemployment rate can lower the real GDP growth. The negative relationship between the change in share prices and the real GDP growth might be caused by the time horizon, as the relationship between the change in share prices and the real GDP growth might be averaged out in the longer run (three months in our case). However, the firm conclusions cannot be reached as these variables are statistically insignificant. It might also be interesting to see the relationships among these variables using data from shorter time horizons, e.g., monthly or weekly series where we leave them for future study.

The across-country structural coefficients from $\hat{\Lambda}_{0}$ in Table 4 indicate the strong influence of shock transmission among G-7 (except the shocks from US, UK, and Canada to Italy, and from UK, Canada, and France to Japan). This result reaffirms the strong economic ties among them as the leading economies.

\section{Concluding Remarks}

In this paper, we propose a new approach to analyze multiple VAR models that result in a newly constructed MtAR. The MtAR construction is based on a matrix-variate 
normal distribution with two covariance matrices. The MtAR is a generalization of VAR

models where the two covariance matrices allow the extension of MtAR to a structural MtAR analysis. The proposed MtAR can also incorporate different lag orders across VAR systems that provide more flexibility to the model. The estimation results from a simulation study and an empirical study on multicountry macroeconomic system show favorable performance of our proposed models and method. The extension of MtAR can cover a wide range of specifications, e.g., time-varying parameters.

\section{Acknowledgements}

We are grateful to the Editor-in-Chief, the Associate Editor and the Reviewer for their helpful feedback that improved the manuscript. We also thank Marco Reale for helpful advice on structural vector autoregressive models.

\section{References}

[1] Abadir, K.M., Magnus, J.R. (2005). Matrix Algebra. New York: Cambridge University Press.

[2] Bańbura, M., Giannone, D., Reichlin, L. (2010). Large Bayesian vector auto regressions. Journal of Applied Econometrics. 25:71-92.

[3] Canova, F., Ciccarelli, M. (2009). Estimating multicountry var models. International Economic Review. 50:929-959.

[4] Canova, F., De Nicolo, G. (2000). Stock returns, term structure, real activity and inflation: An international perspective. Macroeconomic Dynamics. 4:343-372.

[5] Canova, F., Marrinan, J. (1998). Sources and propagations of output shocks: Common shocks or transmission? Journal of International Economics. 42:133-167. 
[6] Chib, S. (2008). Markov chain Monte Carlo methods. The New Palgrave Dictionary of Economics, Second edition. 5:331-338.

[7] Christiano, L.J., Eichenbaum, M., Evans, C.L. (1999). Monetary policy shocks: what have we learned and to what end? In: Taylor, J.B., Woodford, M., eds. Handbook of Macroeconomics, Vol. 1, Chapter 2 (pp. 65-148). Amsterdam: Elsevier.

[8] Christiano, L.J., Eichenbaum, M., Evans, C.L. (2005). Nominal rigidities and the dynamic effects of a shock to monetary policy. Journal of Political Economy. 113:145.

[9] Dawid, A.P. (1981). Some matrix-variate distribution theory: Notational consideration and a Bayesian application. Biometrika. 68:265-274.

[10] Del Negro, M., Obiols, F. (2001). Has monetary policy been so bad that it is better to get rid of it: The case of Mexico. Journal of Money, Credit and Banking. 33:404-433.

[11] Del Negro, M., Schorfheide, F. (2011). Bayesian macroeconometrics. In: Geweke, J., Koop, G., van Dijk, H., eds. The Oxford Handbook of Bayesian Econometrics. New York: Oxford University Press.

[12] Doan, T., Litterman, R.B., Sims, C. (1984). Forecasting and conditional projection using realistic prior distributions. Econometric Reviews. 3:1-144.

[13] Dutilleul, P. (1999). The MLE algorithm for the matrix normal distribution. Journal of Statistical Computation and Simulation. 64:105-123.

[14] Elliott, G., Fatás, A. (1996). International business cycles and the dynamics of the current account. European Economic Review. 40:361-387.

[15] Favero, C.A. (2013). Modelling and forecasting government bond spreads in the euro area: A GVAR model. Journal of Econometrics. 177:343-356. 
[16] Greene, W.H. (2003). Econometric Analysis, Fifth edition. New Jersey: Prentice Hall.

[17] Gupta, A.K., Nagar, D.K. (1999). Matrix Variate Distributions. Florida: Chapman \& Hall/CRC.

[18] Karlsson, S. (2013). Forecasting with Bayesian vector autoregression. In: Elliott, G., Timmermann, A., eds. Handbook of Economic Forecasting, Vol. 2B. Amsterdam: Elsvier.

[19] Koop, G., Korobilis, D. (2009). Bayesian multivariate time series methods for empirical macroeconomics. Foundations and Trends in Econometrics. 3:267-358.

[20] Litterman, R.B. (1980). A Bayesian Procedure for Forecasting with Vector Autoregressions. mimeo. Massachusetts Institute of Technology.

[21] Liu, F. (2012). New Kronecker product decompositions and its applications. Research Inventy: International Journal of Engineering and Science. 1:25-30.

[22] Lütkepohl, H. (2007). New Introduction to Multiple Time Series Analysis. Berlin: Springer.

[23] Rubio-Ramírez, J.F., Waggoner, D.F., Zha, T. (2010). Structural vector autoregressions: Theory of identification and algorithms for inference. The Review of Economic Studies. 77:665-696.

[24] Sims, C.A. (1980). Macroeconomics and reality. Econometrica. 48:1-48.

[25] Sims, C.A., Zha, T. (1998). Bayesian methods for dynamic multivariate models. International Economic Review. 39:949-968.

[26] Stock, J.H., Watson, M.W. (2001). Vector autoregressions. Journal of Economic Perspectives. 15:101-116. 
[27] Watson, M.W. (1994). Business-cycle durations and postwar stabilization of the U.S. economy. American Economic Review. 84:24-46.

[28] Wooldridge, J.M. (2001). Econometric Analysis of Cross Section and Panel Data. Massachusetts: MIT Press. 
TABLE 1

Estimation Results from $2 \times 2$ SMtAR Models

\begin{tabular}{|c|c|c|c|c|c|c|c|c|c|}
\hline \multirow[b]{2}{*}{ Parameter } & \multicolumn{4}{|c|}{$\mathrm{MCMC}$} & \multicolumn{3}{|c|}{ FGLS } & \multicolumn{2}{|c|}{ MLE } \\
\hline & MAD & MAPE* & Avg. S.E. & Avg. SIF & $\mathrm{MAD}^{* *}$ & MAPE* & Avg. S.E.** & $\mathrm{MAD}^{* *}$ & MAPE* \\
\hline \multicolumn{10}{|c|}{$2 \times 2 \operatorname{SMtAR}(1)$} \\
\hline$\hat{\mathbf{B}}$ & 0.0437 & 10.88 & 0.0391 & 2.23 & 0.0460 & 11.41 & 0.0393 & 0.0460 & 11.41 \\
\hline$\hat{\boldsymbol{\Sigma}}$ & 0.0441 & 8.98 & 0.2127 & 2.33 & n.a. & n.a. & n.a. & 0.0944 & 18.04 \\
\hline$\hat{\Psi}$ & 0.1187 & 14.83 & 0.3765 & 2.30 & n.a. & n.a. & n.a. & 0.1456 & 18.22 \\
\hline$\hat{\Omega}$ & 0.0160 & 4.03 & 0.0351 & 2.41 & 0.0226 & 4.99 & n.a. & n.a. & n.a. \\
\hline \multicolumn{10}{|c|}{$2 \times 2 \operatorname{SMtAR}(3)$} \\
\hline$\hat{\mathbf{B}}$ & 0.0480 & n.a. & 0.0460 & 2.41 & 0.0495 & n.a. & 0.0463 & 0.0495 & n.a. \\
\hline$\hat{\Sigma}$ & 0.0198 & 3.62 & 0.2153 & 2.40 & n.a. & n.a. & n.a. & 0.0605 & 10.87 \\
\hline$\hat{\Psi}$ & 0.0528 & 6.83 & 0.3273 & 2.44 & n.a. & n.a. & n.a. & 0.1454 & 18.42 \\
\hline$\hat{\Omega}$ & 0.0336 & 8.66 & 0.0383 & 2.10 & 0.0587 & 14.36 & n.a. & n.a. & n.a. \\
\hline
\end{tabular}

Notes: $\quad$ MCMC iterations $=31,000$ with 1,000 burn-ins and thin $=100$

n.a. $=$ not available

${ }^{*}$ Unavailable MAPEs for $\hat{\mathbf{B}}$ due to some zero-valued true parameters

** $\hat{\boldsymbol{\Sigma}}, \hat{\mathbf{\Psi}}$, and the S.E. of $\hat{\boldsymbol{\Omega}}$ are not estimated under the FGLS.

** $\hat{\Omega}$ is not estimated under the MLE.

TABLE 2

Estimation Results from $3 \times 4$ SMtAR Models

\begin{tabular}{|c|c|c|c|c|c|c|c|c|c|}
\hline \multirow[b]{2}{*}{ Parameter } & \multicolumn{4}{|c|}{ MCMC } & \multicolumn{3}{|c|}{ FGLS } & \multicolumn{2}{|c|}{ MLE } \\
\hline & MAD & MAPE* & Avg. S.E. & Avg. SIF & MAD & MAPE* $^{*}$ & Avg. S.E. & MAD & MAPE* $^{*}$ \\
\hline \multicolumn{10}{|c|}{$3 \times 4 \operatorname{SMtAR}(1)$} \\
\hline$\hat{\mathbf{B}}$ & 0.0615 & n.a. & 0.0743 & 2.31 & 0.0669 & n.a. & 0.0742 & 0.0669 & n.a. \\
\hline$\hat{\Sigma}$ & 0.0182 & 20.22 & 0.0103 & 2.38 & n.a. & n.a. & n.a. & 0.0476 & 23.97 \\
\hline$\hat{\Psi}$ & 0.0413 & 11.05 & 0.0264 & 2.28 & n.a. & n.a. & n.a. & 0.1319 & 34.95 \\
\hline$\hat{\Omega}$ & 0.0072 & 36.90 & 0.0104 & 2.35 & 0.0106 & 43.43 & n.a. & n.a. & n.a. \\
\hline \multicolumn{10}{|c|}{$3 \times 4 \operatorname{SMtAR}(3)$} \\
\hline$\hat{\mathbf{B}}$ & 0.1037 & n.a. & 0.1081 & 2.36 & 0.1078 & n.a. & 0.1161 & 0.1078 & n.a. \\
\hline$\hat{\Sigma}$ & 0.0183 & 16.76 & 0.0099 & 2.35 & n.a. & n.a. & n.a. & 0.0487 & 28.02 \\
\hline$\hat{\Psi}$ & 0.0395 & 12.99 & 0.0256 & 2.30 & n.a. & n.a. & n.a. & 0.1275 & 28.91 \\
\hline$\hat{\Omega}$ & 0.0070 & 36.24 & 0.0107 & 2.48 & 0.0206 & 52.50 & n.a. & n.a. & n.a. \\
\hline \multicolumn{10}{|c|}{$3 \times 4 \operatorname{SMtAR}(\mathbf{p})$} \\
\hline$\hat{\mathbf{B}}$ & 0.0471 & n.a. & 0.0895 & 2.26 & 0.0480 & n.a. & 0.0843 & 0.0480 & n.a. \\
\hline$\hat{\Sigma}$ & 0.0249 & 14.75 & 0.0099 & 2.41 & n.a. & n.a. & n.a. & 0.0478 & 22.85 \\
\hline$\hat{\Psi}$ & 0.0318 & 13.26 & 0.0269 & 2.14 & n.a. & n.a. & n.a. & 0.1374 & 41.40 \\
\hline$\hat{\Omega}$ & 0.0092 & 45.59 & 0.0104 & 2.31 & 0.0092 & 47.66 & n.a. & n.a. & n.a. \\
\hline
\end{tabular}

Notes: $\quad$ MCMC iterations $=26,000$ with 1,000 burn-ins and thin $=50$

n.a. $=$ not available

${ }^{*}$ Unavailable MAPEs for $\hat{\mathbf{B}}$ due to some zero-valued true parameters

** $\hat{\boldsymbol{\Sigma}}, \hat{\mathbf{\Psi}}$, and the S.E. of $\hat{\boldsymbol{\Omega}}$ are not estimated under the FGLS.

** $\hat{\boldsymbol{\Omega}}$ is not estimated under the MLE. 
TABLE 3

Posterior Means and Standard Errors for $\hat{\boldsymbol{\Sigma}}$ and $\hat{A}_{0}$

\begin{tabular}{|c|c|c|c|c|c|}
\hline$\hat{\Sigma}$ & $\% \triangle$ interest & inflation & $\% \triangle$ unemployment & $\% \triangle$ real GDP & $\% \triangle$ share \\
\hline \multirow[t]{2}{*}{$\% \triangle$ interest } & $20.442^{*}$ & 0.051 & -0.648 & 0.104 & -0.259 \\
\hline & $(2.301)$ & $(0.072)$ & $(0.483)$ & $(0.105)$ & $(0.951)$ \\
\hline \multirow[t]{2}{*}{ inflation } & 0.051 & $0.101^{*}$ & 0.022 & -0.002 & $0.120^{*}$ \\
\hline & $(0.072)$ & $(0.009)$ & $(0.035)$ & $(0.007)$ & $(0.062)$ \\
\hline \multirow[t]{2}{*}{$\% \triangle$ unemployment } & -0.648 & 0.022 & $3.995^{*}$ & -0.086 & 0.020 \\
\hline & $(0.483)$ & $(0.035)$ & $(0.450)$ & $(0.047)$ & $(0.423)$ \\
\hline \multirow[t]{2}{*}{$\% \triangle$ real GDP } & 0.104 & -0.002 & -0.086 & $0.185^{*}$ & 0.084 \\
\hline & $(0.105)$ & $(0.007)$ & $(0.047)$ & $(0.018)$ & $(0.088)$ \\
\hline \multirow[t]{2}{*}{$\% \triangle$ share } & -0.259 & $0.120^{*}$ & 0.020 & 0.084 & $14.418^{*}$ \\
\hline & $(0.951)$ & $(0.062)$ & $(0.423)$ & $(0.088)$ & $(1.597)$ \\
\hline$\hat{A}_{0}$ & $\% \triangle$ interest & inflation & $\% \triangle$ unemployment & $\% \triangle$ real GDP & $\% \triangle$ share \\
\hline \multirow[t]{2}{*}{$\% \triangle$ interest } & $0.224^{*}$ & & & & \\
\hline & $(0.013)$ & & & & \\
\hline \multirow[t]{2}{*}{ inflation } & -0.128 & $3.181^{*}$ & & & \\
\hline & $(0.157)$ & $(0.144)$ & & & \\
\hline \multirow[t]{2}{*}{$\% \triangle$ unemployment } & 0.035 & -0.017 & $0.507^{*}$ & & \\
\hline & $(0.027)$ & $(0.028)$ & $(0.028)$ & & \\
\hline \multirow[t]{2}{*}{$\% \triangle$ real GDP } & -0.113 & 0.031 & 0.236 & $2.339^{*}$ & \\
\hline & $(0.128)$ & $(0.124)$ & $(0.127)$ & $(0.115)$ & \\
\hline \multirow[t]{2}{*}{$\% \triangle$ share } & 0.006 & $-0.027^{*}$ & -0.002 & -0.014 & $0.265^{*}$ \\
\hline & $(0.015)$ & $(0.013)$ & $(0.015)$ & $(0.014)$ & $(0.014)$ \\
\hline
\end{tabular}

Notes: Number in parenthesis is standard error.

* denotes statistical significance at $5 \%$. 


\section{TABLE 4}

Posterior Means and Standard Errors for $\hat{\Psi}$ and $\hat{\Lambda}_{0}$

\begin{tabular}{|c|c|c|c|c|c|c|c|}
\hline$\hat{\Psi}$ & US & UK & Canada & France & Germany & Italy & Japan \\
\hline \multirow[t]{2}{*}{ US } & $2.909^{*}$ & $1.533^{*}$ & $1.627^{*}$ & $1.292^{*}$ & $1.590^{*}$ & $1.022^{*}$ & $1.688^{*}$ \\
\hline & $(0.327)$ & $(0.217)$ & $(0.223)$ & $(0.197)$ & $(0.229)$ & $(0.207)$ & $(0.321)$ \\
\hline \multirow[t]{2}{*}{ UK } & $1.533^{*}$ & $2.437^{*}$ & $1.167^{*}$ & $1.405^{*}$ & $1.438^{*}$ & $0.971^{*}$ & $1.119^{*}$ \\
\hline & $(0.217)$ & $(0.266)$ & $(0.182)$ & $(0.194)$ & (0.209) & $(0.194)$ & $(0.278)$ \\
\hline \multirow[t]{2}{*}{ Canada } & $1.627^{*}$ & $1.167^{*}$ & $2.546^{*}$ & $1.217^{*}$ & $1.423^{*}$ & $1.046^{*}$ & $1.328^{*}$ \\
\hline & $(0.223)$ & $(0.182)$ & $(0.270)$ & $(0.183)$ & $(0.209)$ & $(0.199)$ & $(0.278)$ \\
\hline \multirow[t]{2}{*}{ France } & $1.292^{*}$ & $1.405^{*}$ & $1.217^{*}$ & $2.350^{*}$ & $1.773^{*}$ & $1.590^{*}$ & $1.373^{*}$ \\
\hline & $(0.197)$ & $(0.194)$ & $(0.183)$ & $(0.259)$ & $(0.235)$ & $(0.229)$ & $(0.286)$ \\
\hline \multirow[t]{2}{*}{ Germany } & $1.590^{*}$ & $1.438^{*}$ & $1.423^{*}$ & $1.773^{*}$ & $3.078^{*}$ & $1.568^{*}$ & $1.937^{*}$ \\
\hline & $(0.229)$ & $(0.209)$ & $(0.209)$ & $(0.235)$ & $(0.334)$ & $(0.238)$ & $(0.336)$ \\
\hline \multirow[t]{2}{*}{ Italy } & $1.022^{*}$ & $0.971^{*}$ & $1.046^{*}$ & $1.590^{*}$ & $1.568^{*}$ & $3.156^{*}$ & $1.539^{*}$ \\
\hline & $(0.207)$ & $(0.194)$ & $(0.199)$ & $(0.229)$ & $(0.238)$ & $(0.358)$ & $(0.311)$ \\
\hline \multirow[t]{2}{*}{ Japan } & $1.688^{*}$ & $1.119^{*}$ & $1.328^{*}$ & $1.373^{*}$ & $1.937^{*}$ & $1.539^{*}$ & $7.969^{*}$ \\
\hline & $(0.321)$ & $(0.278)$ & $(0.278)$ & $(0.286)$ & $(0.336)$ & $(0.311)$ & $(0.922)$ \\
\hline$\hat{\Lambda}_{0}$ & US & UK & Canada & France & Germany & Italy & Japan \\
\hline \multirow[t]{2}{*}{ US } & $0.844^{*}$ & & & & & & \\
\hline & $(0.046)$ & & & & & & \\
\hline \multirow[t]{2}{*}{ UK } & $-0.278^{*}$ & $0.843^{*}$ & & & & & \\
\hline & $(0.055)$ & $(0.047)$ & & & & & \\
\hline \multirow[t]{2}{*}{ Canada } & $-0.309^{*}$ & $-0.157^{*}$ & $0.765^{*}$ & & & & \\
\hline & $(0.051)$ & $(0.047)$ & $(0.039)$ & & & & \\
\hline \multirow[t]{2}{*}{ France } & -0.023 & $-0.337^{*}$ & $-0.203^{*}$ & $0.952^{*}$ & & & \\
\hline & $(0.065)$ & $(0.067)$ & $(0.059)$ & $(0.054)$ & & & \\
\hline \multirow[t]{2}{*}{ Germany } & $-0.121^{*}$ & $-0.141^{*}$ & $-0.194^{*}$ & $-0.400^{*}$ & $0.694^{*}$ & & \\
\hline & $(0.050)$ & $(0.054)$ & $(0.046)$ & $(0.050)$ & $(0.036)$ & & \\
\hline \multirow[t]{2}{*}{ Italy } & 0.014 & 0.036 & -0.036 & $-0.275^{*}$ & $-0.290^{*}$ & $0.595^{*}$ & \\
\hline & $(0.044)$ & $(0.046)$ & $(0.045)$ & $(0.046)$ & $(0.039)$ & $(0.035)$ & \\
\hline \multirow[t]{2}{*}{ Japan } & $-0.058^{*}$ & -0.007 & -0.038 & -0.014 & $-0.113^{*}$ & $-0.115^{*}$ & $0.356^{*}$ \\
\hline & $(0.023)$ & $(0.023)$ & $(0.022)$ & $(0.024)$ & $(0.023)$ & $(0.022)$ & $(0.020)$ \\
\hline
\end{tabular}

Notes: Number in parenthesis is standard error.

* denotes statistical significance at $5 \%$. 


\section{A Selected Results from Some SMtAR Models}

TABLE A1

\begin{tabular}{|c|c|c|c|c|c|}
\hline \multicolumn{6}{|c|}{ Estimated Regression Coefficients $(\hat{\mathbf{B}})$} \\
\hline \multirow{2}{*}{$\begin{array}{l}2 \times 2 \operatorname{SMtAR}(1) \\
\text { Parameter }\end{array}$} & \multirow[b]{2}{*}{ T.V. } & \multicolumn{2}{|c|}{ MCMC } & \multicolumn{2}{|c|}{ FGLS } \\
\hline & & Mean & S.E. & Mean & S.E. \\
\hline \multicolumn{6}{|l|}{$c^{1}$} \\
\hline$c_{1}^{1}$ & 0.5 & 0.39 & 0.06 & 0.38 & 0.06 \\
\hline$c_{2}^{1}$ & -0.7 & -0.78 & 0.04 & -0.79 & 0.04 \\
\hline \multicolumn{6}{|l|}{$\overline{A_{1}^{1}}$} \\
\hline$a_{11, t-1}^{1}$ & 0.5 & 0.51 & 0.03 & 0.51 & 0.03 \\
\hline$a_{21, t-1}^{1}$ & 0.4 & 0.42 & 0.02 & 0.43 & 0.02 \\
\hline$a_{12, t-1}^{1}$ & 0.1 & 0.09 & 0.03 & 0.09 & 0.03 \\
\hline$a_{22, t-1}^{1}$ & 0.5 & 0.49 & 0.02 & 0.48 & 0.02 \\
\hline \multicolumn{6}{|l|}{$c^{2}$} \\
\hline$c_{1}^{2}$ & -0.9 & -0.80 & 0.08 & -0.80 & 0.08 \\
\hline$c_{2}^{2}$ & 1 & 1.08 & 0.05 & 1.09 & 0.06 \\
\hline \multicolumn{6}{|l|}{$A_{1}^{2}$} \\
\hline$a_{11, t-1}^{2}$ & 0.1 & 0.13 & 0.03 & 0.13 & 0.03 \\
\hline$a_{21, t-1}^{2}$ & 0.2 & 0.24 & 0.02 & 0.24 & 0.03 \\
\hline$a_{12, t-1}^{2}$ & 0.3 & 0.31 & 0.04 & 0.31 & 0.04 \\
\hline$a_{22, t-1}^{2}$ & 0.3 & 0.29 & 0.03 & 0.29 & 0.03 \\
\hline \multicolumn{2}{|l|}{$3 \times 4 \operatorname{SMtAR}(1)$} & \multicolumn{2}{|c|}{ MCMC } & \multicolumn{2}{|c|}{ FGLS } \\
\hline Parameter & T.V. & Mean & S.E. & Mean & S.E. \\
\hline \multicolumn{6}{|l|}{$c^{1}$} \\
\hline$c_{1}^{1}$ & 0.5 & 0.48 & 0.02 & 0.49 & 0.01 \\
\hline$c_{2}^{1}$ & -0.7 & -0.66 & 0.07 & -0.67 & 0.07 \\
\hline$c_{3}^{1}$ & 0.1 & -0.10 & 0.14 & -0.12 & 0.14 \\
\hline \multicolumn{6}{|l|}{$A_{1}^{1}$} \\
\hline$a_{11, t-1}^{1}$ & 0.5 & 0.53 & 0.02 & 0.52 & 0.01 \\
\hline$a_{21, t-1}^{1}$ & 0.4 & 0.34 & 0.07 & 0.34 & 0.07 \\
\hline$a_{31, t-1}^{1}$ & 0.25 & 0.48 & 0.14 & 0.51 & 0.14 \\
\hline$a_{12, t-1}^{1}$ & 0.1 & 0.11 & 0.01 & 0.11 & 0.01 \\
\hline$a_{22, t-1}^{1}$ & 0.2 & 0.15 & 0.03 & 0.15 & 0.03 \\
\hline$a_{32, t-1}^{1}$ & 0.25 & 0.34 & 0.06 & 0.33 & 0.06 \\
\hline$a_{13, t-1}^{1}$ & 0.05 & 0.05 & 0.00 & 0.05 & 0.00 \\
\hline$a_{23, t-1}^{1}$ & 0.1 & 0.10 & 0.02 & 0.10 & 0.01 \\
\hline$a_{33, t-1}^{1}$ & 0 & -0.03 & 0.03 & -0.04 & 0.03 \\
\hline \multicolumn{6}{|l|}{$c^{2}$} \\
\hline$c_{1}^{2}$ & -0.9 & -0.91 & 0.01 & -0.91 & 0.01 \\
\hline$c_{2}^{2}$ & 1 & 1.01 & 0.04 & 1.02 & 0.04 \\
\hline$c_{3}^{2}$ & 0.6 & 0.54 & 0.08 & 0.55 & 0.08 \\
\hline
\end{tabular}


TABLE A1

(Continued)

\begin{tabular}{|c|c|c|c|c|c|}
\hline \multicolumn{2}{|c|}{$3 \times 4 \operatorname{SMtAR}(1)$} & \multicolumn{2}{|c|}{$\mathrm{MCMC}$} & \multicolumn{2}{|c|}{ FGLS } \\
\hline Parameter & T.V. & Mean & S.E. & Mean & S.E. \\
\hline \multicolumn{6}{|l|}{$A_{1}^{2}$} \\
\hline$a_{11, t-1}^{2}$ & 0.1 & 0.11 & 0.01 & 0.11 & 0.01 \\
\hline$a_{21, t-1}^{2}$ & 0.2 & 0.15 & 0.03 & 0.15 & 0.03 \\
\hline$a_{31, t-1}^{2}$ & 0.25 & 0.28 & 0.06 & 0.29 & 0.06 \\
\hline$a_{12, t-1}^{2}$ & 0.3 & 0.31 & 0.01 & 0.31 & 0.01 \\
\hline$a_{22, t-1}^{2}$ & 0.3 & 0.28 & 0.03 & 0.27 & 0.03 \\
\hline$a_{32, t-1}^{2}$ & 0.2 & 0.24 & 0.05 & 0.24 & 0.05 \\
\hline$a_{13, t-1}^{2}$ & 0.2 & 0.20 & 0.00 & 0.20 & 0.00 \\
\hline$a_{23, t-1}^{2}$ & 0 & 0.00 & 0.01 & 0.00 & 0.01 \\
\hline$a_{33, t-1}^{2}$ & 0.1 & 0.09 & 0.03 & 0.09 & 0.03 \\
\hline \multicolumn{6}{|l|}{$c^{3}$} \\
\hline$c_{1}^{3}$ & -0.5 & -0.51 & 0.11 & -0.53 & 0.07 \\
\hline$c_{2}^{3}$ & 0.6 & 0.34 & 0.37 & 0.33 & 0.38 \\
\hline$c_{3}^{3}$ & 0.7 & 0.96 & 0.62 & 0.98 & 0.72 \\
\hline \multicolumn{6}{|l|}{$A_{1}^{3}$} \\
\hline$a_{11, t-1}^{3}$ & 0.9 & 0.90 & 0.02 & 0.89 & 0.01 \\
\hline$a_{21, t-1}^{3}$ & 0.4 & 0.37 & 0.06 & 0.37 & 0.06 \\
\hline$a_{31, t-1}^{3}$ & 0.6 & 0.65 & 0.09 & 0.66 & 0.11 \\
\hline$a_{12, t-1}^{3}$ & 0.05 & 0.05 & 0.01 & 0.05 & 0.01 \\
\hline$a_{22, t-1}^{3}$ & 0.3 & 0.28 & 0.03 & 0.27 & 0.03 \\
\hline$a_{32, t-1}^{3}$ & 0.2 & 0.15 & 0.06 & 0.15 & 0.06 \\
\hline$a_{13, t-1}^{3}$ & 0 & 0.00 & 0.00 & 0.00 & 0.00 \\
\hline$a_{23, t-1}^{3}$ & 0.1 & 0.10 & 0.02 & 0.10 & 0.02 \\
\hline$a_{33, t-1}^{3}$ & 0.05 & 0.06 & 0.03 & 0.06 & 0.03 \\
\hline \multicolumn{6}{|l|}{$c^{4}$} \\
\hline$c_{1}^{4}$ & 1 & 1.00 & 0.07 & 0.93 & 0.05 \\
\hline$c_{2}^{4}$ & 0.2 & 0.56 & 0.25 & 0.58 & 0.25 \\
\hline$c_{3}^{4}$ & -0.1 & -0.44 & 0.47 & -0.43 & 0.47 \\
\hline \multicolumn{6}{|l|}{$A_{1}^{4}$} \\
\hline$a_{11, t-1}^{4}$ & 0.6 & 0.61 & 0.02 & 0.62 & 0.01 \\
\hline$a_{21, t-1}^{4}$ & 0.3 & 0.20 & 0.07 & 0.19 & 0.07 \\
\hline$a_{31, t-1}^{4}$ & 0.25 & 0.32 & 0.13 & 0.32 & 0.13 \\
\hline$a_{12, t-1}^{4}$ & 0.2 & 0.20 & 0.01 & 0.20 & 0.01 \\
\hline$a_{22, t-1}^{4}$ & 0.1 & 0.09 & 0.04 & 0.09 & 0.04 \\
\hline$a_{32, t-1}^{4}$ & 0.25 & 0.30 & 0.07 & 0.30 & 0.07 \\
\hline$a_{13, t-1}^{4}$ & 0.1 & 0.10 & 0.00 & 0.10 & 0.00 \\
\hline$a_{23, t-1}^{4}$ & 0 & 0.01 & 0.02 & 0.01 & 0.02 \\
\hline$a_{33, t-1}^{4}$ & 0.25 & 0.25 & 0.03 & 0.25 & 0.04 \\
\hline
\end{tabular}


TABLE A1

(Continued)

\begin{tabular}{|c|c|c|c|c|c|}
\hline \multirow{2}{*}{$\begin{array}{l}3 \times 4 \mathrm{SMtAR}(\mathbf{p}) \\
\text { Parameter }\end{array}$} & \multirow[b]{2}{*}{ T.V. } & \multicolumn{2}{|c|}{ MCMC } & \multicolumn{2}{|c|}{ FGLS } \\
\hline & & Mean & S.E. & Mean & S.E. \\
\hline \multicolumn{6}{|l|}{$c^{1}$} \\
\hline$c_{1}^{1}$ & 0.5 & 0.48 & 0.02 & 0.47 & 0.02 \\
\hline$c_{2}^{1}$ & -0.7 & -0.63 & 0.08 & -0.63 & 0.08 \\
\hline$c_{3}^{1}$ & 0.1 & 0.21 & 0.16 & 0.20 & 0.15 \\
\hline \multicolumn{6}{|l|}{$A_{1}^{1}$} \\
\hline$a_{11, t-1}^{1}$ & 0.5 & 0.52 & 0.02 & 0.53 & 0.02 \\
\hline$a_{21, t-1}^{1}$ & 0.4 & 0.32 & 0.08 & 0.32 & 0.07 \\
\hline$a_{31, t-1}^{1}$ & 0.25 & 0.09 & 0.15 & 0.11 & 0.15 \\
\hline$a_{12, t-1}^{1}$ & 0.1 & 0.10 & 0.01 & 0.10 & 0.01 \\
\hline$a_{22, t-1}^{1}$ & 0.2 & 0.21 & 0.03 & 0.21 & 0.03 \\
\hline$a_{32, t-1}^{1}$ & 0.25 & 0.25 & 0.06 & 0.26 & 0.06 \\
\hline$a_{13, t-1}^{1}$ & 0.05 & 0.05 & 0.00 & 0.05 & 0.00 \\
\hline$a_{23, t-1}^{1}$ & 0.1 & 0.11 & 0.02 & 0.10 & 0.02 \\
\hline$a_{33, t-1}^{1}$ & 0 & -0.05 & 0.03 & -0.06 & 0.03 \\
\hline \multicolumn{6}{|l|}{$c^{2}$} \\
\hline$c_{1}^{2}$ & -0.9 & -0.90 & 0.03 & -0.90 & 0.02 \\
\hline$c_{2}^{2}$ & 1 & 1.08 & 0.08 & 1.08 & 0.08 \\
\hline$c_{3}^{2}$ & 0.6 & 0.65 & 0.16 & 0.64 & 0.16 \\
\hline \multicolumn{6}{|l|}{$A_{1}^{2}$} \\
\hline$a_{11, t-1}^{2}$ & 0.1 & 0.11 & 0.04 & 0.12 & 0.02 \\
\hline$a_{21, t-1}^{2}$ & 0.2 & 0.24 & 0.09 & 0.24 & 0.09 \\
\hline$a_{31, t-1}^{2}$ & 0.25 & 0.09 & 0.18 & 0.09 & 0.18 \\
\hline$a_{12, t-1}^{2}$ & 0.3 & 0.30 & 0.01 & 0.30 & 0.01 \\
\hline$a_{22, t-1}^{2}$ & 0.3 & 0.33 & 0.03 & 0.34 & 0.03 \\
\hline$a_{32, t-1}^{2}$ & 0.2 & 0.20 & 0.05 & 0.20 & 0.05 \\
\hline$a_{13, t-1}^{2}$ & 0.2 & 0.20 & 0.00 & 0.20 & 0.00 \\
\hline$a_{23, t-1}^{2}$ & 0 & -0.01 & 0.02 & -0.02 & 0.01 \\
\hline$a_{33, t-1}^{2}$ & 0.1 & 0.05 & 0.03 & 0.04 & 0.03 \\
\hline \multicolumn{6}{|l|}{$\frac{33, t-1}{A_{2}^{2}}$} \\
\hline$a_{11, t-2}^{2}$ & 0.05 & 0.04 & 0.02 & 0.04 & 0.01 \\
\hline$a_{21, t-2}^{2}$ & 0.1 & 0.08 & 0.04 & 0.07 & 0.04 \\
\hline$a_{31, t-2}^{2}$ & 0.05 & 0.15 & 0.07 & 0.15 & 0.07 \\
\hline$a_{12, t-2}^{2}$ & 0.1 & 0.10 & 0.02 & 0.10 & 0.01 \\
\hline$a_{22, t-2}^{2}$ & 0.2 & 0.16 & 0.03 & 0.16 & 0.03 \\
\hline$a_{32, t-2}^{2}$ & 0.1 & 0.12 & 0.07 & 0.13 & 0.07 \\
\hline$a_{13, t-2}^{2}$ & 0.05 & 0.05 & 0.01 & 0.05 & 0.00 \\
\hline$a_{23, t-2}^{2}$ & 0.1 & 0.09 & 0.02 & 0.09 & 0.02 \\
\hline$a_{33, t-2}^{2}$ & 0.2 & 0.22 & 0.03 & 0.22 & 0.03 \\
\hline \multicolumn{6}{|l|}{$c^{3}$} \\
\hline$c_{1}^{3}$ & -0.5 & -0.45 & 0.17 & -0.51 & 0.10 \\
\hline$c_{2}^{3}$ & 0.6 & 0.49 & 0.54 & 0.40 & 0.50 \\
\hline$c_{3}^{3}$ & 0.7 & 0.08 & 1.00 & 0.00 & 0.99 \\
\hline
\end{tabular}


TABLE A1

(Continued)

\begin{tabular}{|c|c|c|c|c|c|}
\hline \multirow{2}{*}{$\begin{array}{l}3 \times 4 \mathrm{SMtAR}(\mathbf{p}) \\
\text { Parameter }\end{array}$} & \multirow[b]{2}{*}{ T.V. } & \multicolumn{2}{|c|}{$\mathrm{MCMC}$} & \multicolumn{2}{|c|}{ FGLS } \\
\hline & & Mean & S.E. & Mean & S.E. \\
\hline \multicolumn{6}{|l|}{$A_{1}^{3}$} \\
\hline$a_{11, t-1}^{3}$ & 0.9 & 0.86 & 0.05 & 0.89 & 0.03 \\
\hline$a_{21, t-1}^{3}$ & 0.4 & 0.37 & 0.17 & 0.38 & 0.16 \\
\hline$a_{31, t-1}^{3}$ & 0.6 & 0.47 & 0.31 & 0.48 & 0.31 \\
\hline$a_{12, t-1}^{3}$ & 0.05 & 0.05 & 0.01 & 0.05 & 0.01 \\
\hline$a_{22, t-1}^{3}$ & 0.3 & 0.31 & 0.04 & 0.31 & 0.04 \\
\hline$a_{32, t-1}^{3}$ & 0.2 & 0.25 & 0.08 & 0.25 & 0.07 \\
\hline$a_{13, t-1}^{3}$ & 0 & 0.00 & 0.01 & 0.00 & 0.00 \\
\hline$a_{23, t-1}^{3}$ & 0.1 & 0.09 & 0.02 & 0.09 & 0.02 \\
\hline$a_{33, t-1}^{3}$ & 0.05 & 0.04 & 0.03 & 0.04 & 0.03 \\
\hline \multicolumn{6}{|l|}{$A_{2}^{3}$} \\
\hline$a_{11, t-2}^{3}$ & 0.01 & 0.03 & 0.07 & -0.02 & 0.04 \\
\hline$a_{21, t-2}^{3}$ & 0.05 & 0.16 & 0.22 & 0.14 & 0.21 \\
\hline$a_{31, t-2}^{3}$ & 0 & -0.09 & 0.41 & -0.10 & 0.41 \\
\hline$a_{12, t-2}^{3}$ & 0 & 0.01 & 0.01 & 0.00 & 0.01 \\
\hline$a_{22, t-2}^{3}$ & 0.05 & 0.01 & 0.04 & 0.01 & 0.04 \\
\hline$a_{32, t-2}^{3}$ & 0.1 & 0.03 & 0.07 & 0.03 & 0.07 \\
\hline$a_{13, t-2}^{3}$ & 0 & 0.00 & 0.00 & -0.01 & 0.00 \\
\hline$a_{23, t-2}^{3}$ & 0.05 & 0.07 & 0.02 & 0.07 & 0.02 \\
\hline$a_{33, t-2}^{3}$ & 0.01 & 0.00 & 0.03 & 0.00 & 0.03 \\
\hline \multicolumn{6}{|l|}{$A_{3}^{3}$} \\
\hline$a_{11, t-3}^{3}$ & 0 & 0.03 & 0.05 & 0.04 & 0.03 \\
\hline$a_{21, t-3}^{3}$ & 0 & -0.10 & 0.16 & -0.09 & 0.15 \\
\hline$a_{31, t-3}^{3}$ & 0 & 0.16 & 0.31 & 0.16 & 0.29 \\
\hline$a_{12, t-3}^{3}$ & 0 & 0.00 & 0.01 & 0.00 & 0.01 \\
\hline$a_{22, t-3}^{3}$ & 0 & 0.02 & 0.03 & 0.02 & 0.03 \\
\hline$a_{32, t-3}^{3}$ & 0.01 & 0.03 & 0.06 & 0.03 & 0.06 \\
\hline$a_{13, t-3}^{3}$ & 0 & 0.00 & 0.00 & 0.00 & 0.00 \\
\hline$a_{23, t-3}^{3}$ & 0 & 0.01 & 0.02 & 0.00 & 0.01 \\
\hline$a_{33, t-3}^{3}$ & 0 & 0.01 & 0.03 & 0.00 & 0.03 \\
\hline \multicolumn{6}{|l|}{$c^{4}$} \\
\hline$c_{1}^{4}$ & 1 & 0.99 & 0.06 & 0.98 & 0.04 \\
\hline$c_{2}^{4}$ & 0.2 & 0.19 & 0.21 & 0.20 & 0.22 \\
\hline$c_{3}^{4}$ & -0.1 & -0.42 & 0.43 & -0.40 & 0.44 \\
\hline \multicolumn{6}{|l|}{$A_{1}^{4}$} \\
\hline$a_{11, t-1}^{4}$ & 0.6 & 0.61 & 0.02 & 0.61 & 0.01 \\
\hline$a_{21, t-1}^{4}$ & 0.3 & 0.29 & 0.06 & 0.28 & 0.06 \\
\hline$a_{31, t-1}^{4}$ & 0.25 & 0.32 & 0.12 & 0.32 & 0.12 \\
\hline$a_{12, t-1}^{4}$ & 0.2 & 0.20 & 0.01 & 0.20 & 0.01 \\
\hline$a_{22, t-1}^{4}$ & 0.1 & 0.12 & 0.04 & 0.12 & 0.03 \\
\hline$a_{32, t-1}^{4}$ & 0.25 & 0.33 & 0.07 & 0.33 & 0.07 \\
\hline$a_{13, t-1}^{4}$ & 0.1 & 0.10 & 0.00 & 0.10 & 0.00 \\
\hline$a_{23, t-1}^{4}$ & 0 & 0.00 & 0.02 & 0.00 & 0.02 \\
\hline$a_{33, t-1}^{4}$ & 0.25 & 0.23 & 0.03 & 0.23 & 0.04 \\
\hline
\end{tabular}


TABLE A2

Estimated Across-row Covariance Coefficients $(\hat{\boldsymbol{\Sigma}})$

\begin{tabular}{|c|c|c|c|c|}
\hline \multirow{2}{*}{$\begin{array}{l}2 \times 2 \text { SMtAR }(1) \\
\text { Parameter }\end{array}$} & \multirow[b]{2}{*}{ T.V. } & \multicolumn{2}{|c|}{ MCMC } & \multirow{2}{*}{$\begin{array}{l}\text { MLE } \\
\text { Mean }\end{array}$} \\
\hline & & Mean & S.E. & \\
\hline$\sigma_{11}$ & 1 & 0.9266 & 0.3866 & 0.8362 \\
\hline$\sigma_{21}$ & 0.3536 & 0.3057 & 0.1293 & 0.2751 \\
\hline$\sigma_{12}$ & 0.3536 & 0.3057 & 0.1293 & 0.2751 \\
\hline$\sigma_{22}$ & 0.5 & 0.4927 & 0.2055 & 0.4432 \\
\hline \multicolumn{2}{|l|}{$3 \times 4 \operatorname{SMtAR}(1)$} & \multicolumn{2}{|c|}{$\mathrm{MCMC}$} & MLE \\
\hline Parameter & T.V. & Mean & S.E. & Mean \\
\hline$\sigma_{11}$ & 0.01 & 0.0159 & 0.0007 & 0.0075 \\
\hline$\sigma_{21}$ & -0.025 & -0.0228 & 0.0018 & -0.0182 \\
\hline$\sigma_{31}$ & 0.01 & 0.0131 & 0.0027 & 0.0113 \\
\hline$\sigma_{12}$ & -0.025 & -0.0228 & 0.0018 & -0.0182 \\
\hline$\sigma_{22}$ & 0.25 & 0.2328 & 0.0119 & 0.1840 \\
\hline$\sigma_{32}$ & 0.15 & 0.1292 & 0.0124 & 0.1061 \\
\hline$\sigma_{13}$ & 0.01 & 0.0131 & 0.0027 & 0.0113 \\
\hline$\sigma_{23}$ & 0.15 & 0.1292 & 0.0124 & 0.1061 \\
\hline$\sigma_{33}$ & 1 & 0.9119 & 0.0460 & 0.7441 \\
\hline \multicolumn{2}{|l|}{$3 \times 4 \operatorname{SMtAR}(\mathbf{p})$} & \multicolumn{2}{|c|}{ MCMC } & MLE \\
\hline Parameter & T.V. & Mean & S.E. & Mean \\
\hline$\sigma_{11}$ & 0.01 & 0.0157 & 0.0007 & 0.0078 \\
\hline$\sigma_{21}$ & -0.025 & -0.0230 & 0.0017 & -0.0199 \\
\hline$\sigma_{31}$ & 0.01 & 0.0100 & 0.0027 & 0.0083 \\
\hline$\sigma_{12}$ & -0.025 & -0.0230 & 0.0017 & -0.0199 \\
\hline$\sigma_{22}$ & 0.25 & 0.2312 & 0.0120 & 0.1904 \\
\hline$\sigma_{32}$ & 0.15 & 0.1216 & 0.0116 & 0.1059 \\
\hline$\sigma_{13}$ & 0.01 & 0.0100 & 0.0027 & 0.0083 \\
\hline$\sigma_{23}$ & 0.15 & 0.1216 & 0.0116 & 0.1059 \\
\hline$\sigma_{33}$ & 1 & 0.8609 & 0.0441 & 0.7334 \\
\hline
\end{tabular}


TABLE A3

Estimated Across-column Covariance Coefficients $(\hat{\mathbf{\Psi}})$

\begin{tabular}{|c|c|c|c|c|}
\hline \multirow{2}{*}{$\begin{array}{l}2 \times 2 \text { SMtAR }(1) \\
\text { Parameter }\end{array}$} & \multirow[b]{2}{*}{ T.V. } & \multicolumn{2}{|c|}{ MCMC } & \multirow{2}{*}{$\begin{array}{l}\text { MLE } \\
\text { Mean }\end{array}$} \\
\hline & & Mean & S.E. & \\
\hline$\psi_{11}$ & 0.7 & 0.8014 & 0.3277 & 0.8271 \\
\hline$\psi_{21}$ & -0.753 & -0.8672 & 0.3550 & -0.8929 \\
\hline$\psi_{12}$ & -0.753 & -0.8672 & 0.3550 & -0.8929 \\
\hline$\psi_{22}$ & 1 & 1.1449 & 0.4685 & 1.1755 \\
\hline \multicolumn{2}{|l|}{$3 \times 4 \operatorname{SMtAR}(1)$} & \multicolumn{2}{|c|}{$\mathrm{MCMC}$} & MLE \\
\hline Parameter & T.V. & Mean & S.E. & Mean \\
\hline$\psi_{11}$ & 1 & 0.9103 & 0.0491 & 1.3315 \\
\hline$\psi_{21}$ & 0.6364 & 0.5570 & 0.0317 & 0.8511 \\
\hline$\psi_{31}$ & 0.2324 & 0.1890 & 0.0211 & 0.2916 \\
\hline$\psi_{41}$ & 0.0949 & 0.0864 & 0.0236 & 0.1398 \\
\hline$\psi_{12}$ & 0.6364 & 0.5570 & 0.0317 & 0.8511 \\
\hline$\psi_{22}$ & 0.5 & 0.4757 & 0.0249 & 0.6728 \\
\hline$\psi_{32}$ & 0.2739 & 0.2349 & 0.0178 & 0.3552 \\
\hline$\psi_{42}$ & 0.1342 & 0.1237 & 0.0181 & 0.1932 \\
\hline$\psi_{13}$ & 0.2324 & 0.1890 & 0.0211 & 0.2916 \\
\hline$\psi_{23}$ & 0.2739 & 0.2349 & 0.0178 & 0.3552 \\
\hline$\psi_{33}$ & 0.6 & 0.5835 & 0.0323 & 0.8202 \\
\hline$\psi_{43}$ & -0.2939 & -0.2563 & 0.0232 & -0.3865 \\
\hline$\psi_{14}$ & 0.0949 & 0.0864 & 0.0236 & 0.1398 \\
\hline$\psi_{24}$ & 0.1342 & 0.1237 & 0.0181 & 0.1932 \\
\hline$\psi_{34}$ & -0.2939 & -0.2563 & 0.0232 & -0.3865 \\
\hline$\psi_{44}$ & 0.9 & 0.8070 & 0.0456 & 1.1824 \\
\hline \multicolumn{2}{|l|}{$3 \times 4 \operatorname{SMtAR}(\mathbf{p})$} & \multicolumn{2}{|c|}{ MCMC } & MLE \\
\hline Parameter & T.V. & Mean & S.E. & Mean \\
\hline$\psi_{11}$ & 1 & 0.9902 & 0.0540 & 1.3696 \\
\hline$\psi_{21}$ & 0.6364 & 0.6025 & 0.0342 & 0.8669 \\
\hline$\psi_{31}$ & 0.2324 & 0.1910 & 0.0201 & 0.2749 \\
\hline$\psi_{41}$ & 0.0949 & 0.1235 & 0.0245 & 0.1808 \\
\hline$\psi_{12}$ & 0.6364 & 0.6025 & 0.0342 & 0.8669 \\
\hline$\psi_{22}$ & 0.5 & 0.5019 & 0.0265 & 0.6759 \\
\hline$\psi_{32}$ & 0.2739 & 0.2244 & 0.0169 & 0.3260 \\
\hline$\psi_{42}$ & 0.1342 & 0.1571 & 0.0189 & 0.2292 \\
\hline$\psi_{13}$ & 0.2324 & 0.1910 & 0.0201 & 0.2749 \\
\hline$\psi_{23}$ & 0.2739 & 0.2244 & 0.0169 & 0.3260 \\
\hline$\psi_{33}$ & 0.6 & 0.5397 & 0.0289 & 0.7346 \\
\hline$\psi_{43}$ & -0.2939 & -0.2618 & 0.0222 & -0.3852 \\
\hline$\psi_{14}$ & 0.0949 & 0.1235 & 0.0245 & 0.1808 \\
\hline$\psi_{24}$ & 0.1342 & 0.1571 & 0.0189 & 0.2292 \\
\hline$\psi_{34}$ & -0.2939 & -0.2618 & 0.0222 & -0.3852 \\
\hline$\psi_{44}$ & 0.9 & 0.8801 & 0.0480 & 1.2234 \\
\hline
\end{tabular}

